\title{
Frustrated pentagonal Cairo lattice in the non-collinear antiferromagnet $\mathrm{Bi}_{4} \mathrm{Fe}_{5} \mathrm{O}_{13} \mathrm{~F}$
}

\author{
Artem M. Abakumov, ${ }^{1, *}$ Dmitry Batuk, ${ }^{1}$ Alexander A. Tsirlin, ${ }^{2,3, \dagger}$ Clemens Prescher, ${ }^{4}$ Leonid Dubrovinsky, ${ }^{4}$ \\ Denis V. Sheptyakov, ${ }^{5}$ Walter Schnelle, ${ }^{2}$ Joke Hadermann, ${ }^{1}$ and Gustaaf Van Tendeloo ${ }^{1}$ \\ ${ }^{1}$ EMAT, University of Antwerp, Groenenborgerlaan 171, B-2020 Antwerp, Belgium \\ ${ }^{2}$ Max Planck Institute for Chemical Physics of Solids, Nöthnitzer Str. 40, 01187 Dresden, Germany \\ ${ }^{3}$ National Institute of Chemical Physics and Biophysics, 12618 Tallinn, Estonia \\ ${ }^{4}$ Bayerisches Geoinstitut, Universität Bayreuth, 95440 Bayreuth, Germany \\ ${ }^{5}$ Laboratory for Neutron Scattering, Paul Scherrer Institut, CH-5232 Villigen, Switzerland
}

(Received 9 October 2012; revised manuscript received 27 November 2012; published 30 January 2013)

\begin{abstract}
We report on the crystal structure and magnetism of the iron-based oxyfluoride $\mathrm{Bi}_{4} \mathrm{Fe}_{5} \mathrm{O}_{13} \mathrm{~F}$, a material prototype of the Cairo pentagonal spin lattice. The crystal structure of $\mathrm{Bi}_{4} \mathrm{Fe}_{5} \mathrm{O}_{13} \mathrm{~F}$ is determined by a combination of neutron diffraction, synchrotron x-ray diffraction, and transmission electron microscopy. It comprises layers of $\mathrm{FeO}_{6}$ octahedra and $\mathrm{FeO}_{4}$ tetrahedra forming deformed pentagonal units. The topology of these layers resembles a pentagonal least-perimeter tiling, which is known as the Cairo lattice. This topology gives rise to frustrated exchange couplings and underlies a sequence of magnetic transitions at $T_{1}=62 \mathrm{~K}, T_{2}=71 \mathrm{~K}$, and $T_{N}=178 \mathrm{~K}$, as determined by thermodynamic measurements and neutron diffraction. Below $T_{1}, \mathrm{Bi}_{4} \mathrm{Fe}_{5} \mathrm{O}_{13} \mathrm{~F}$ forms a fully ordered non-collinear antiferromagnetic structure, whereas the magnetic state between $T_{1}$ and $T_{N}$ may be partially disordered according to the sizable increase in the magnetic entropy at $T_{1}$ and $T_{2} . \mathrm{Bi}_{4} \mathrm{Fe}_{5} \mathrm{O}_{13} \mathrm{~F}$ reveals unanticipated magnetic transitions on the pentagonal Cairo spin lattice and calls for a further work on finite-temperature properties of this strongly frustrated spin model.
\end{abstract}

DOI: 10.1103/PhysRevB.87.024423

PACS number(s): 75.25.-j, 75.50.Ee, 61.66.Fn, 75.30.Et

\section{INTRODUCTION}

Magnetically frustrated systems entail a pattern of interactions that cannot be satisfied simultaneously. ${ }^{1,2}$ These patterns typically involve equivalent antiferromagnetic (AFM) interactions arranged in closed loops with an odd number of bonds, as in a pentagon or in a triangle. As one moves around the loop, the spin flips on each bond, and the translation symmetry is violated because the full turn around the loop leads to the inverted spin direction. Therefore, in frustrated systems spins cannot develop a simple collinear AFM order; instead more complex magnetic ground states appear. ${ }^{2}$ Finding appropriate model systems for experimental studies of frustrated magnetism is a long-standing challenge. ${ }^{3-6}$ The availability of model materials is crucial for verifying existing theoretical concepts and expanding the family of frustrated spin systems.

The loops with an odd number of bonds are typically triangular. Planar triangular spin lattices have been widely studied $^{1,2,7,8}$ in attempts to achieve a spin-liquid state that lacks long-range magnetic order down to zero temperature. When triangles form corner-sharing tetrahedra instead of tiling the plane, the system enters a so-called spin-ice state that facilitates experimental access to magnetic monopoles. $1,2,6,9$ Thanks to a different topology, spin lattices based on pentagons could lead to a range of hitherto unexplored properties and ground states. However, experimental and even theoretical considerations of such lattices remain challenging because the five-fold symmetry of an ideal pentagon excludes the periodicity of the lattice.

Pentagonal units do not form a regular tiling in the plane. To build a tiling, the pentagons have to be distorted and should, unlike the triangles, involve nonequivalent bonds. One of the least-perimeter tilings allowed for pentagons is known as the Cairo lattice. It entails the coupling $J_{1}$ between three-vertex sites, and the coupling $J_{2}$ running from each three-vertex site to the surrounding four-vertex sites (Fig. 1). Recent theoretical work puts forward a number of unconventional ground states, including spin nematics and other interesting properties of the Cairo spin lattice. ${ }^{10-12}$ These predictions remain highly challenging for experimental verification. In crystals, the pentagonal arrangement of magnetic ions is very rare, which is partly related to the fact that periodic crystals cannot have five-fold symmetry. Presently, the only known material prototype of the Cairo lattice is $\mathrm{Bi}_{2} \mathrm{Fe}_{4} \mathrm{O}_{9}$ having a mullite-type crystal structure. ${ }^{13-15}$

Here, we present the crystal structure, magnetic structure, and thermodynamic properties of the material $\mathrm{Bi}_{4} \mathrm{Fe}_{5} \mathrm{O}_{13} \mathrm{~F}$. We show that this compound entails a pentagonal arrangement of the spin- $\frac{5}{2} \mathrm{Fe}^{3+}$ ions forming the Cairo-type spin lattice. While the magnetic ground state of $\mathrm{Bi}_{4} \mathrm{Fe}_{5} \mathrm{O}_{13} \mathrm{~F}$ is non-collinear and follows expectations for the Cairo spin lattice, the behavior at finite temperatures and the sequence of magnetic transitions are highly unusual and so far unexpected by theory.

The outline of this paper is as follows: In Sec. II, experimental and computational methods are reported. In Sec. III, we present a comprehensive crystallographic investigation and refine the crystal structure of $\mathrm{Bi}_{4} \mathrm{Fe}_{5} \mathrm{O}_{13}$ both above and below the magnetic transitions (Sec. III A). We further study the magnetic ground state (Sec. III B), report thermodynamic properties (Sec. III C), and develop a microscopic magnetic model that accounts for main features of the experimental data (Sec. III D). We conclude our study with a brief discussion and summary (Sec. IV).

\section{METHODS}

Powder samples of $\mathrm{Bi}_{4} \mathrm{Fe}_{5} \mathrm{O}_{13} \mathrm{~F}$ were prepared by a solidstate reaction of $\mathrm{Bi}_{2} \mathrm{O}_{3}$ (99.9\% Aldrich), $\mathrm{Fe}_{2} \mathrm{O}_{3}$ (nanopowder $<50 \mathrm{~nm}$, Aldrich) and $\mathrm{BiF}_{3}(99.99 \%$ Aldrich). The initial 

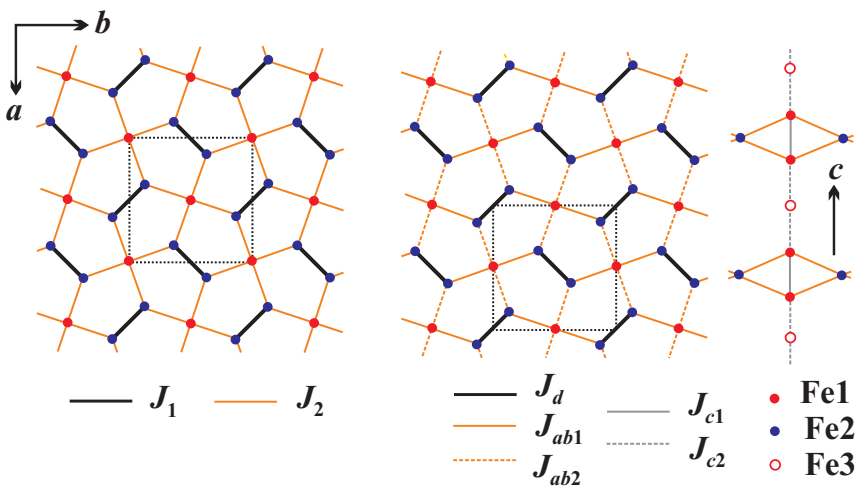

FIG. 1. (Color online) Cairo pentagonal lattice (left panel) and its extended version in $\mathrm{Bi}_{4} \mathrm{Fe}_{5} \mathrm{O}_{13} \mathrm{~F}$ (right panel). Note that the ideal lattice comprises only two couplings, $J_{1}$ and $J_{2}$, whereas in $\mathrm{Bi}_{4} \mathrm{Fe}_{5} \mathrm{O}_{13} \mathrm{~F} J_{2}$ splits into $J_{a b 1}$ and $J_{a b 2}$. Additionally, the couplings $J_{c 1}$ and $J_{c 2}$ along the $c$ direction are present.

compounds were weighed in stoichiometric ratios, mixed, rigorously ground, and pressed into pellets. The pellets were placed into alumina crucibles, covered with a lid, and then sealed in quartz tubes under a dynamic vacuum of $10^{-3}$ mbar. The samples were annealed at $650{ }^{\circ} \mathrm{C}$ for $3 \mathrm{~h}$ and at $750{ }^{\circ} \mathrm{C}$ for $6 \mathrm{~h}$ with intermediate regrinding.

X-ray powder diffraction (XRPD) measurements were conducted with a Huber G670 Guinier diffractometer $\mathrm{Cu}$ $\mathrm{K}_{\alpha 1}$ radiation, curved $\mathrm{Ge}(111)$ monochromator, transmission mode, image plate). High-resolution synchrotron x-ray powder diffraction (SXPD) data were collected at the ID31 beamline of the European Synchrotron Radiation Facility (ESRF, Grenoble, France) using a wavelength of $0.40006 \AA$ and eight scintillation detectors, each preceded by a $\mathrm{Si}(111)$ analyzer crystal. The powder sample was contained in a thin-walled quartz capillary that was spun during the experiment. The temperature of the sample was controlled by a He-flow cryostat (temperature range $10-300 \mathrm{~K}$ ) and hot-air blower (300-700 K).

Neutron powder diffraction (NPD) data were collected with the high-resolution powder diffractometer HRPT [Paul Scherrer Institute (PSI), Switzerland] at the wavelength of $1.8857 \AA$ with the use of a standard orange cryostat and radiation-type furnace to cover the temperature range from 1.5 to $700 \mathrm{~K}$. Structure solution was performed with direct methods using the EXPO software. ${ }^{16}$ The Rietveld refinement of the crystal and magnetic structure against the powder diffraction data was performed with the JANA2006 package. ${ }^{17}$

Transmission electron microscopy (TEM) investigation was performed with a FEI Tecnai $\mathrm{G}^{2}$ microscope operated at $200 \mathrm{kV}$. The TEM specimen was prepared by grinding the sample in ethanol and depositing the dispersion on a copper grid covered with a holey carbon film. The sample was analyzed by means of electron diffraction (ED) and high-resolution high-angle annular dark field scanning transmission electron microscopy (HAADF-STEM). The simulated HAADF-STEM images were calculated using the QSTEM 2.0 software. ${ }^{18}$ The $\mathrm{Bi}$ and $\mathrm{Fe}$ content has been measured by energy dispersive $\mathrm{x}$-ray (EDX) analysis performed with a Jeol JEM5510 scanning electron microscope equipped with an INCA EDX system (Oxford instruments). More than 50 spectra have been collected in order to obtain a statistically relevant result. Within the experimental error, the measured $\mathrm{Bi} / \mathrm{Fe}=0.84(6)$ ratio agrees with the nominal chemical composition.

The magnetic susceptibility of $\mathrm{Bi}_{4} \mathrm{Fe}_{5} \mathrm{O}_{13} \mathrm{~F}$ was measured with a Quantum Design magnetic properties measurement system (MPMS) superconducting quantum interference device (SQUID) magnetometer in the temperature range 2-600 K in magnetic fields up to $5 \mathrm{~T}$. Heat capacity was measured by a relaxation technique using a Quantum Design physical properties measurement system (PPMS) in the temperature range $1.8-320 \mathrm{~K}$.

The Mössbauer spectrum was recorded at room temperature $(293 \mathrm{~K})$ in transmission mode using a constant-acceleration Mössbauer spectrometer with a nominal $2.02 \mathrm{GBq}^{57} \mathrm{Co}$ source in a $6 \mu \mathrm{m} \mathrm{Rh}$ matrix (conventional source). The velocity scale was calibrated relative to a $30 \mu \mathrm{m} \alpha$-Fe foil using the positions certified for the National Bureau of Standards standard reference material no. 1541; a line width of $0.30 \mathrm{~mm} / \mathrm{s}$ for the outer lines of $\alpha$-Fe was obtained at room temperature. The spectrum collection time was 10 days. The Mössbauer spectrum was fit using the MOSSA software package. ${ }^{19}$

The electronic structure of $\mathrm{Bi}_{4} \mathrm{Fe}_{5} \mathrm{O}_{13} \mathrm{~F}$ was calculated within the density functional theory (DFT) framework using the FPLO $\operatorname{code}^{20}$ and the Perdew-Wang parametrization of the exchange-correlation potential. ${ }^{21}$ The symmetry-irreducible part of the first Brillouin zone was sampled with a $k$ mesh of 72 points for the unit cell with 92 atoms. Strong correlations in the Fe $3 d$ shell were treated by the DFT $+U$ procedure with a Coulomb repulsion $U=7 \mathrm{eV}$ and Hund's exchange $J=$ $1 \mathrm{eV} .^{22}$ Magnetic couplings were evaluated by mapping total energies of collinear spin configurations onto the Heisenberg model:

$$
\hat{H}=\sum_{\langle i j\rangle} J_{i j} \mathbf{S}_{i} \mathbf{S}_{j},
$$

where the summation is over all bonds $\langle i j\rangle$, and exchange integrals $J_{i j}$ are the coupling energies per bond.

\section{RESULTS}

\section{A. Crystal structure}

$\mathrm{X}$-ray power diffraction investigation reveals a singlephase $\mathrm{Bi}_{4} \mathrm{Fe}_{5} \mathrm{O}_{13} \mathrm{~F}$ material with a tetragonal unit cell with lattice parameters $a=8.30318$ (5) $\AA, c=18.0640$ (1) $\AA$, and the space group $P 4_{2} / m b c$ or its acentric subgroup $P 4_{2} b c$. The electron diffraction patterns of $\mathrm{Bi}_{4} \mathrm{Fe}_{5} \mathrm{O}_{13} \mathrm{~F}$ (Fig. 2) confirm the unit cell and space symmetry. The structure solution was performed in the centrosymmetric $P 4_{2} / m b c$ space group with direct methods providing the positions of all cations and part of the anions in the unit cell. The missing anions were located using difference Fourier maps. The resulting structural model was refined against the SXPD data at the temperatures of 10, 298, and $673 \mathrm{~K}$ and against the NPD data at temperatures of $1.5,300$, and $700 \mathrm{~K} .{ }^{23}$ A magnetic contribution was taken into account for the refinement of the crystal structure from the NPD data collected at $T=1.5 \mathrm{~K}$ (see details in Sec. III B). Oxygen and fluorine anions cannot be distinguished reliably from either $\mathrm{x}$-ray or neutron powder diffraction. Therefore, the 

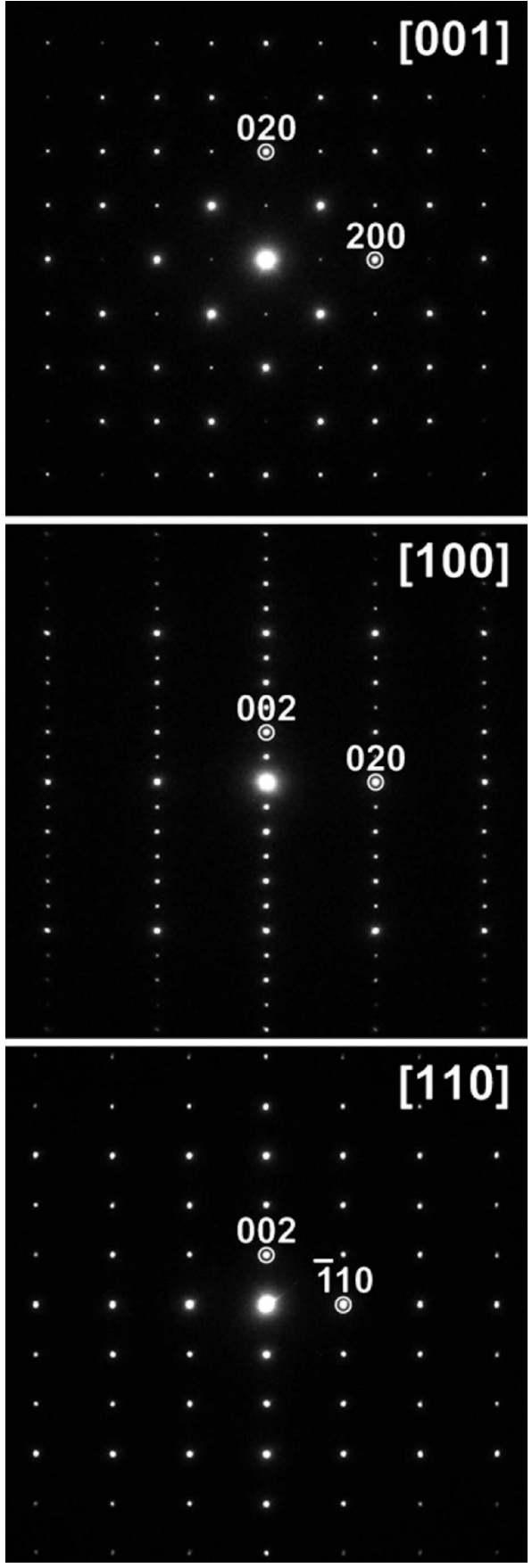

FIG. 2. ED patterns of $\mathrm{Bi}_{4} \mathrm{Fe}_{5} \mathrm{O}_{13} \mathrm{~F}$ along main zone axes.

assignment of the fluorine position at $4 b\left(0,0, \frac{1}{4}\right)$ is based on bond-valence-sum (BVS) arguments.

Bond-valence-sum arguments strongly favor a complete ordering of the $\mathrm{O}$ and $\mathrm{F}$ anions. The $\mathrm{F}^{-}$anions linked to four $\mathrm{Bi}$ cations acquire a BVS of 0.91 (2), which is close to their nominal valence. For the $\mathrm{O}^{2-}$ anions occupying this position, the BVS would be as low as 1.20 (2), thus demonstrating a strong and unrealistic underbonding. The anion ordering is additionally supported by the room-temperature Mössbauer spectrum that is consistent with only two distinct coordination types of $\mathrm{Fe}^{3+}$ cations (see below).

The refinement of the low-temperature diffraction data resulted in low atomic displacements parameters (ADPs) for all atomic positions. However, at and above room temperature high ADPs of F1 and O1 indicated possible displacements of these atoms. Indeed, the displacements of $\mathrm{F} 1$ and $\mathrm{O} 1$ from their special positions reduced the ADPs. This effect is mostly of dynamic nature, because at low temperatures the displacements are suppressed. The $\mathrm{O} 1$ and $\mathrm{F} 1$ displacements observed at high temperatures may be driven by subtle adjustments of interatomic distances and coordination (see BVS analysis in the Supplemental Material ${ }^{23}$ ), although further studies of the local structure would be necessary to fully elucidate this issue. We have also checked the possibility of symmetry lowering. The refinement in the acentric group $\mathrm{P}_{2} b c$ neither eliminated the disorder, nor reduced the reliability factors. Electron diffraction patterns clearly demonstrate the presence of glide planes perpendicular to the $a$ and $b$ unit cell axes, as well as perpendicular to the diagonals of the $a b$ face. Therefore, any further symmetry reduction can be safely excluded.

The crystallographic information for $\mathrm{Bi}_{4} \mathrm{Fe}_{5} \mathrm{O}_{13} \mathrm{~F}$ at room temperature is summarized in Tables I and II. Further details on the structure refinement and crystallographic data at $T=1.5$ and $700 \mathrm{~K}$ are given in the Supplemental Material. ${ }^{23}$

The crystal structure of $\mathrm{Bi}_{4} \mathrm{Fe}_{5} \mathrm{O}_{13} \mathrm{~F}$ is illustrated in Figs. 3(a)-3(c). It is built of infinite chains of edge-sharing $\mathrm{FeO}_{6}$ octahedra running along the $c$ axis and comprising two Fe positions: $\mathrm{Fe} 1$ and $\mathrm{Fe} 3$ (Fig. 3). The $\mathrm{Fe} 2$ atoms form pairs of corner-sharing $\mathrm{FeO}_{4}$ tetrahedra that link the octahedral chains into a framework. These links repeat at every third $\mathrm{FeO}_{6}$ octahedron of the chain, thus creating large cavities in the framework. The cavities are occupied by the fluorine-centered tetrahedral $\mathrm{Bi}_{4} \mathrm{~F}$ groups.

The structure of $\mathrm{Bi}_{4} \mathrm{Fe}_{5} \mathrm{O}_{13} \mathrm{~F}$ viewed along the $c$ direction immediately reveals the pentagonal arrangement of the magnetic $\mathrm{Fe}^{3+}$ cations [compare Fig. 3(c) and Fig. 1(a)]. While in $\mathrm{Bi}_{2} \mathrm{Fe}_{4} \mathrm{O}_{9}$ similar pentagonal planes are directly stacked on top of each other, $\mathrm{Bi}_{4} \mathrm{Fe}_{5} \mathrm{O}_{13} \mathrm{~F}$ demonstrates a more peculiar stacking, with the pentagonal planes interleaved by the $\mathrm{Fe}^{3} \mathrm{O}_{6}$ octahedra [Fig. 3(a)].

The $\mathrm{Bi}_{4} \mathrm{Fe}_{5} \mathrm{O}_{13} \mathrm{~F}$ crystal structure has been validated by HAADF-STEM imaging along the [001] and [100] directions (Fig. 4). In HAADF-STEM, the brightness of a dot corresponding to the projection of an atomic column roughly scales as $Z^{n}$ $(1.5<n<2)$, where $Z$ is the average atomic number along the column. Therefore, Bi-bearing atomic columns appear as the brighter dots and form the most prominent contrast in the image. The insets in Fig. 4 show HAADF-STEM images calculated using the refined atomic positions. The good agreement between the experimental and calculated images justifies the structure solution.

The room-temperature Mössbauer spectrum for $\mathrm{Bi}_{4} \mathrm{Fe}_{5} \mathrm{O}_{13} \mathrm{~F}^{23}$ can be decomposed into two paramagnetic doublets, contributing to 57 (4)\% (doublet I) and 43 (4)\% (doublet II) of the total absorption area. Mössbauer parameters, such as center shift (CS) and quadrupole splitting (QS), are as follows:

(1) Doublet I: CS $=0.428(9) \mathrm{mm} / \mathrm{s}, \mathrm{QS}=0.39(2) \mathrm{mm} / \mathrm{s}$;

(2) Doublet II: $\mathrm{CS}=0.19$ (1) $\mathrm{mm} / \mathrm{s}, \mathrm{QS}=0.65$ (2) $\mathrm{mm} / \mathrm{s}$.

The CS values for both doublets provide evidence that all iron is in the $\mathrm{Fe}^{3+}$ state. ${ }^{24,25}$ The CS of the doublet I is consistent with $\mathrm{Fe}^{3+}$ in an octahedral coordination. ${ }^{25}$ The significantly smaller CS of the doublet II is in agreement with 
TABLE I. Atomic positions and displacement parameters $\left(U_{\text {iso }}\right)$ of $\mathrm{Bi}_{4} \mathrm{Fe}_{5} \mathrm{O}_{13} \mathrm{~F}\left[T=300 \mathrm{~K}\right.$, space group $P 4_{2} / m b c, a=8.2995(1) \AA$, $c=18.0573$ (3) $\AA, R_{F}=0.013, R_{P}=0.029$ ].

\begin{tabular}{|c|c|c|c|c|c|}
\hline Atom & Position & $x / a$ & $y / b$ & $z / c$ & $U_{\text {iso }}\left(\AA^{2}\right)$ \\
\hline Bi1 & $16 i$ & $0.6706(2)$ & $0.6592(2)$ & $0.15697(7)$ & $0.0064(3)$ \\
\hline Fe1 & $8 f$ & $\frac{1}{2}$ & 0 & 0.0800 & 0.0049 (4) \\
\hline $\mathrm{Fe} 2$ & $8 h$ & $0.8515(2)$ & $0.8388(2)$ & 0 & 0.0058 (4) \\
\hline $\mathrm{Fe} 3$ & $4 d$ & $\frac{1}{2}$ & 0 & $\frac{1}{4}$ & $0.0037(5)$ \\
\hline O1 & $8 e^{\mathrm{a}}$ & 0 & 0 & $0.0072(6)$ & 0.014 (1) \\
\hline $\mathrm{O} 2$ & $16 i$ & $0.2914(2)$ & $0.8760(3)$ & 0.5858 (1) & $0.0092(5)$ \\
\hline $\mathrm{O} 3$ & $8 h$ & $0.1362(3)$ & $0.5900(3)$ & 0 & $0.0065(6)$ \\
\hline $\mathrm{O} 4$ & $8 g$ & $0.3268(3)$ & $x+\frac{1}{2}$ & $\frac{3}{4}$ & 0.0077 (6) \\
\hline $\mathrm{O} 5$ & $16 i$ & $0.5823(2)$ & $0.8603(2)$ & $0.3331(2)$ & $0.0076(4)$ \\
\hline $\mathrm{F} 1$ & $16 i^{\mathrm{b}}$ & 0.024 & 0 & $0.2597(7)$ & $0.007(5)$ \\
\hline
\end{tabular}

Reduced occupancy factor: $g(\mathrm{O} 1)=\frac{1}{2}$.

${ }^{\mathrm{b}}$ Reduced occupancy factor: $g(\mathrm{~F} 1)=\frac{1}{4}$.

the tetrahedrally coordinated $\mathrm{Fe}^{3+}$ (Ref. 25). The 57 (4):43 (4) ratio of the octahedrally and tetrahedrally coordinated iron corresponds well to the anticipated 60:40 ratio in $\mathrm{Bi}_{4} \mathrm{Fe}_{5} \mathrm{O}_{13} \mathrm{~F}$.

\section{B. Magnetic structure}

Below $T_{N}=178 \mathrm{~K}$, extra reflections appear on the NPD pattern. These reflections originate from a magnetic ordering, as no changes have been seen in the SXPD patterns down to $T=10 \mathrm{~K}$. The magnetic reflections in the entire temperature range of $T=1.5$ to $178 \mathrm{~K}$ can be indexed with a $\mathbf{k}=\left(\frac{1}{2}, \frac{1}{2}, 0\right)$ propagation vector. The magnetic structure at $T=1.5 \mathrm{~K}$ was solved using a tetragonal magnetic supercell $\mathbf{a}_{m}=\mathbf{a}-$ $\mathbf{b}, \mathbf{b}_{m}=\mathbf{a}+\mathbf{b}, \mathbf{c}_{m}=\mathbf{c}$. The analysis of possible magnetic symmetries was performed with the ISODISTORT software. ${ }^{26}$ The solution was found in the magnetic space group $P_{C} 4_{2} / n .^{23}$

The magnetic moments of the $\mathrm{Fe}$ positions, which are symmetrically equivalent in the nuclear structure, were kept identical. Although the symmetry does not require identical magnetic moments for $\mathrm{Fe} 1$ and $\mathrm{Fe} 3$, we found that these moments are equal within the standard deviation. Therefore, the corresponding linear constraint was introduced without affecting the fit quality. The refined magnetic moments at $T=1.5 \mathrm{~K}$ are $4.06(6) \mu_{B}$ for the $\mathrm{Fe} 1$ and $\mathrm{Fe} 3$ positions and

TABLE II. Selected interatomic distances (in $\AA$ ) for $\mathrm{Bi}_{4} \mathrm{Fe}_{5} \mathrm{O}_{13} \mathrm{~F}$ at $300 \mathrm{~K}$

\begin{tabular}{lccc}
\hline \hline Bi1-O2 & $2.173(3)$ & Bi1-F1 & $2.35(1)$ \\
Bi1-O4 & $2.125(2)$ & Bi1-F1 & $2.57(2)$ \\
Bi1-O5 & $2.168(2)$ & Bi1-F1 & $2.58(1)$ \\
Bi1-O5 & $2.555(2)$ & Bi1-F1 & $2.79(2)$ \\
$\mathrm{BVS}=3.15(1)$ & & & \\
$\mathrm{Fe} 1-\mathrm{O} 2 \times 2$ & $2.017(2)$ & $\mathrm{Fe} 2-\mathrm{O} 1$ & $1.824(2)$ \\
$\mathrm{Fe} 1-\mathrm{O} 3 \times 2$ & $1.981(2)$ & $\mathrm{Fe} 2-\mathrm{O} 2 \times 2$ & $1.900(2)$ \\
$\mathrm{Fe} 1-\mathrm{O} 5 \times 2$ & $2.067(3)$ & $\mathrm{Fe} 2-\mathrm{O} 3$ & $1.882(3)$ \\
$\mathrm{BVS}=2.964(8)$ & & $\mathrm{BVS}=2.93(1)$ & \\
$\mathrm{Fe} 3-\mathrm{O} 4 \times 2$ & $2.032(2)$ & & \\
$\mathrm{Fe} 3-\mathrm{O} 5 \times 2$ & $2.015(2)$ & & \\
$\mathrm{BVS}=2.956(7)$ & & & \\
\hline \hline
\end{tabular}

3.34 (6) $\mu_{B}$ for the Fe 2 position. Details on the refined magnetic moment components are listed in Table III.

Experimental values of the magnetic moments are significantly smaller than the expected moment of $5 \mu_{B}$ for $\mathrm{Fe}^{3+}$ in its high-spin configuration. This reduction is very typical for $\mathrm{AFM} \mathrm{Fe}^{3+}$ oxides, because both $\mathrm{Fe}-\mathrm{O}$ hybridization and spin fluctuations in an AFM ground state tend to reduce the observable magnetic moment with respect to its ideal value. For example, magnetic moments of octahedrally coordinated $\mathrm{Fe}^{3+}$ atoms hover around $4 \mu_{B}, 27,28$ whereas tetrahedrally coordinated $\mathrm{Fe}^{3+}$ may have even lower magnetic moments close to $3 \mu_{B} \cdot{ }^{29}$

The peculiar non-collinear antiferromagnetic structure of $\mathrm{Bi}_{4} \mathrm{Fe}_{5} \mathrm{O}_{13} \mathrm{~F}$ is shown in Fig. 5. All magnetic moments are confined to the $a b$ plane. The order along the octahedral chains is ferrimagnetic, with two parallel magnetic moments on the neighboring $\mathrm{Fe} 1$ atoms, and an opposite moment on $\mathrm{Fe} 3$. The direction of these moments is close to [110] and rotates by $90^{\circ}$ between neighboring chains, so that the moments in second-neighbor chains are opposite and cancel each other. The magnetic moments of the $\mathrm{Fe} 2$ atoms are $\sim 4.2^{\circ}$ off the 100 direction and form an AFM configuration within each $\mathrm{Fe}_{2} \mathrm{O}_{7}$ dimer (Fig. 5).

The temperature evolution of the magnetic reflections is nonmonotonic and pinpoints at least two additional magnetic transitions that follow the initial AFM ordering at $T_{N}=178 \mathrm{~K}$ (Fig. 6). The transition at $T_{1} \approx 62 \mathrm{~K}$ is manifested by abrupt intensity variations: the reflections with even $l$ rapidly vanish, whereas the intensities of the reflections with odd $l$ notably increase. The second transition at $T_{2} \approx 71 \mathrm{~K}$ manifests itself by the vanishing of the $\frac{1}{2} 0 l, l=2 n$ reflections. The two magnetic transitions below $T_{N}$ are well in line with the results of thermodynamic measurements (Sec. IIIC). These transitions are likely accompanied by drastic changes in the magnetic structure. Details of this complex and intriguing magnetic behavior should be addressed in future studies.

\section{Thermodynamic properties}

Magnetic susceptibility of $\mathrm{Bi}_{4} \mathrm{Fe}_{5} \mathrm{O}_{13} \mathrm{~F}$ reveals an overall antiferromagnetic behavior (Fig. 7) in agreement with the antiferromagnetic, albeit non-collinear spin arrangement, 

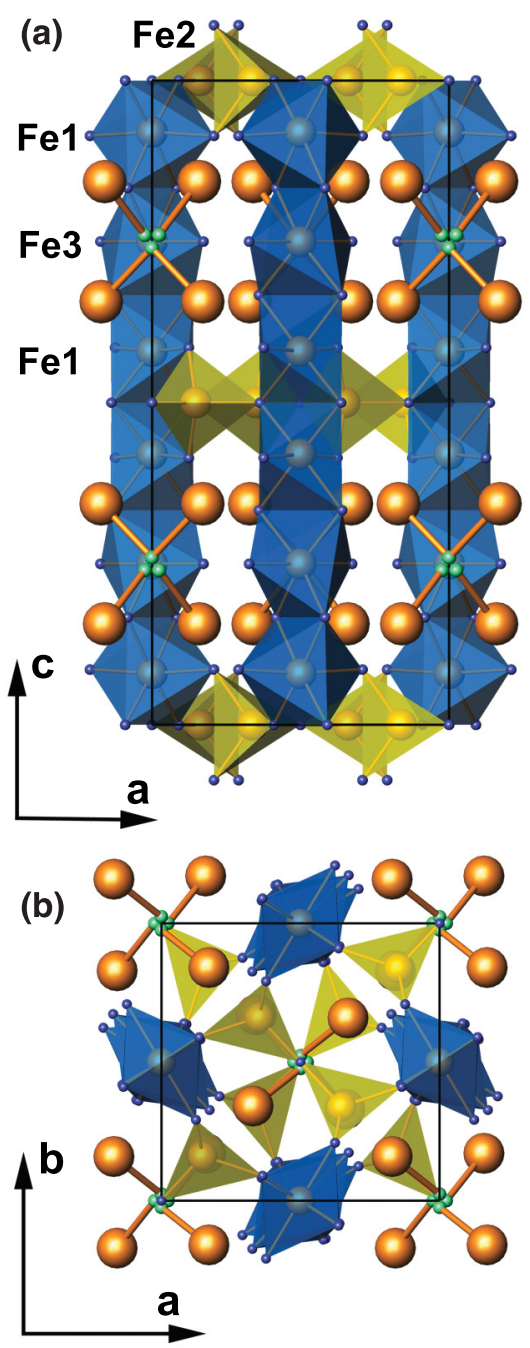

(c)

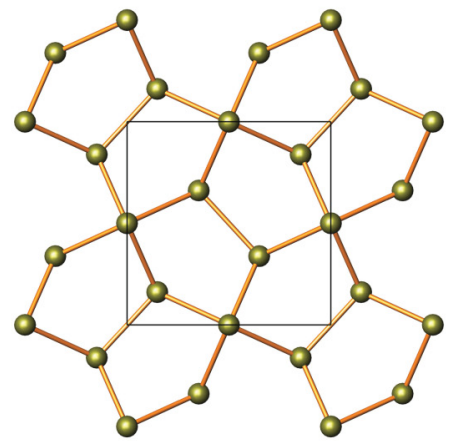

FIG. 3. (Color online) Crystal structure of $\mathrm{Bi}_{4} \mathrm{Fe}_{5} \mathrm{O}_{13} \mathrm{~F}$ : (a) [010] projection, (b) [001] projection, (c) Cairo pentagonal lattice of the $\mathrm{Fe}$ atoms. $\mathrm{FeO}_{6}$ octahedra and $\mathrm{FeO}_{4}$ tetrahedra are shown in blue and yellow, respectively. The $\mathrm{Bi}, \mathrm{F}, \mathrm{Fe}$, and $\mathrm{O}$ atoms are drawn as large orange, small light green, medium green, and small blue spheres, respectively.

pinpointed by neutron diffraction. The field dependence of the susceptibility should be ascribed to a tiny amount of a ferromagnetic impurity. Magnetization loops measured at constant temperature do not show any clear signature of a remnant magnetization or hysteresis (Fig. 8, top).

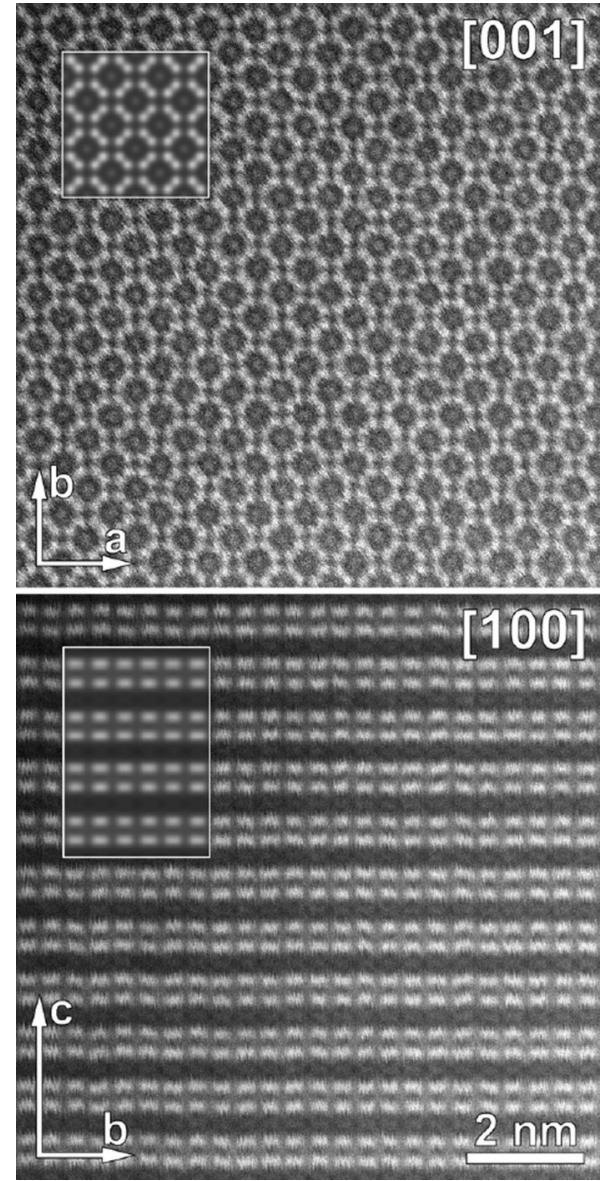

FIG. 4. [001] and [100] HAADF-STEM images of $\mathrm{Bi}_{4} \mathrm{Fe}_{5} \mathrm{O}_{13} \mathrm{~F}$. The insets show the images calculated using the refined crystal structure. The size of the [001] calculated image is $3 a \times 3 b$ (thickness $t=4 \mathrm{~nm}$ ); the size of the [100] calculated image is $3 b \times 2 c$ (thickness $t=7 \mathrm{~nm})$.

Above $300 \mathrm{~K}$, the susceptibility follows the Curie-Weiss law:

$$
\chi=C /(T+\theta),
$$

with $C=3.59(3) \mathrm{emu} \mathrm{K} /(\mathrm{mol} \mathrm{Fe})$ and $\theta=-380(10) \mathrm{K}$ (Fig. 8, bottom). The resulting Curie constant corresponds to an effective magnetic moment of $\mu_{\text {eff }}=5.4 \mu_{B}$, in reasonable agreement with $5.92 \mu_{B}$ expected for the spin-5/2 $\mathrm{Fe}^{3+}$ cations. The negative $\theta$ indicates predominant AFM interactions in $\mathrm{Bi}_{4} \mathrm{Fe}_{5} \mathrm{O}_{13} \mathrm{~F}$.

In fields of $0.1 \mathrm{~T}$ and higher (Fig. 7, bottom part), the most conspicuous effect is the double anomaly at $T_{1}=62 \mathrm{~K}$ and $T_{2}=71 \mathrm{~K}$. In lower fields, a third anomaly around $T_{N}=178 \mathrm{~K}$ could be additionally observed (Fig. 7, top). Heat-capacity $\left(C_{p}\right)$ measurements consistently show the anomalies at $T_{1}, T_{2}$, and $T_{N}$, thus confirming the intrinsic nature of these magnetic transitions (Fig. 7, inset). The anomalies at $T_{1}$ and $T_{2}$ are indicative of first-order transitions with small latent heats.

Considering the neutron diffraction data, we conclude that $\mathrm{Bi}_{4} \mathrm{Fe}_{5} \mathrm{O}_{13} \mathrm{~F}$ undergoes an antiferromagnetic ordering of the $\mathrm{Fe}$ moments at $T_{N}=178 \mathrm{~K}$. However, this ordering is presumably incomplete, as shown by the large anomalies in the heat capacity at $T_{1}$ and $T_{2}$. The area under the heat-capacity curve 
TABLE III. Positions of magnetic atoms, components of their magnetic moments, and total magnetic moments $(M)$ in $\mathrm{Bi}_{4} \mathrm{Fe}_{5} \mathrm{O}_{13} \mathrm{~F}$ at $1.5 \mathrm{~K}$ $\left(a=b=11.7192 \AA, c=18.0501 \AA\right.$, all $\left.m_{z}=0\right)$.

\begin{tabular}{|c|c|c|c|c|c|c|}
\hline Atom & $x / a$ & $y / b$ & $z / c$ & $m_{x}$ & $m_{y}$ & $M\left(\mu_{B}\right)$ \\
\hline $\mathrm{Fe} 1_{1}$ & $\frac{1}{4}$ & $\frac{1}{4}$ & 0.0801 & 4.05 (2) & $-0.35(6)$ & $4.06(6)$ \\
\hline $\mathrm{Fe}_{2}$ & $\frac{1}{4}$ & $\frac{1}{4}$ & 0.4199 & 4.05 (2) & $-0.35(6)$ & $4.06(6)$ \\
\hline $\mathrm{Fe} 2_{1}$ & 0.0063 & 0.8448 & 0 & 2.18 & $-2.53(4)$ & $3.34(6)$ \\
\hline $\mathrm{Fe} 22_{2}$ & 0.0063 & -0.3448 & $\frac{1}{2}$ & $2.53(4)$ & $-2.18(4)$ & $3.34(6)$ \\
\hline $\mathrm{Fe} 3$ & $\frac{1}{4}$ & $\frac{1}{4}$ & $\frac{1}{4}$ & $4.05(2)$ & $0.35(6)$ & $4.06(6)$ \\
\hline
\end{tabular}

plotted as $C_{p} / T$ vs $T$ corresponds to the entropy release upon a magnetic transition. The large amount of entropy released at $T_{1}$ and $T_{2}$ implies that above $T_{1}$ the system develops an incompletely ordered intermediate AFM structure, which is further altered at $T_{2}$ and eventually disappears at $T_{N}$. This peculiar behavior is independently confirmed by the drastic changes in the magnetic neutron scattering (Sec. III B).

\section{Microscopic magnetic model}

To elucidate relevant magnetic interactions, the band structure of $\mathrm{Bi}_{4} \mathrm{Fe}_{5} \mathrm{O}_{13} \mathrm{~F}$ was calculated. Regarding the band structure, this oxyfluoride is very similar to insulating $\mathrm{Fe}^{3+}$ oxides. Its electronic spectrum (Fig. 9) reveals oxygen and fluorine $2 p$ bands between $-5 \mathrm{eV}$ and the Fermi level. The Fe $3 d$ bands are around $-6 \mathrm{eV}$ (filled) and at $+2 \mathrm{eV}$ (empty), with

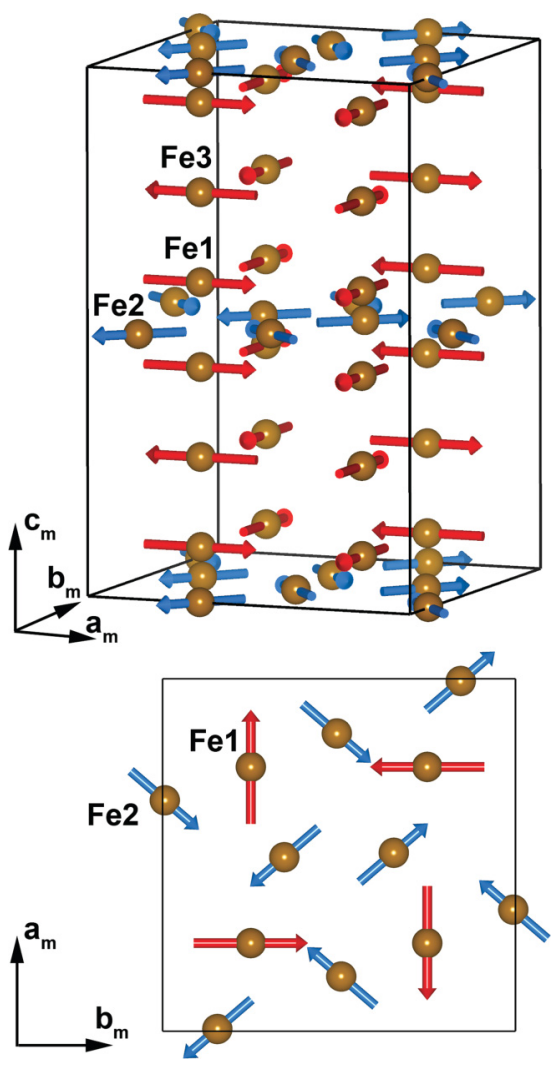

FIG. 5. (Color online) Magnetic structure of $\mathrm{Bi}_{4} \mathrm{Fe}_{5} \mathrm{O}_{13} \mathrm{~F}$ at $T=$ $1.5 \mathrm{~K}$. The orientation of the magnetic moments within the pentagonal $\mathrm{Fe} 1-\mathrm{Fe} 2$ layer at $z=\frac{1}{2}$ is shown at the bottom. The axes denote the magnetic unit cell, which is twice larger than the crystallographic unit cell. the splitting of $8 \mathrm{eV}$ that roughly matches the on-site Coulomb repulsion of $U=7 \mathrm{eV}$. $\mathrm{Bi} 6 s$ orbitals form bonding states at around $-10 \mathrm{eV}$.

In DFT $+U$ calculations, the energy gap of $1.5-2.0 \mathrm{eV}$ depending on the spin configuration concurs with the brown color of $\mathrm{Bi}_{4} \mathrm{Fe}_{5} \mathrm{O}_{13} \mathrm{~F}$. Magnetic moments of $\mathrm{Fe}$ atoms are about 4.6 $\mu_{B}$, which is larger than the experimental values of 4.06 (6) $\mu_{B}$ and 3.34 (6) $\mu_{B}$ for $\mathrm{Fe} 1, \mathrm{Fe} 3$, and Fe2, respectively (see Table III). However, the direct comparison between the DFT $+U$ estimates and the experimental values from neutron diffraction is usually impossible, because DFT $+U$ does not account for the effect of antiferromagnetic spin fluctuations. For example, our reference calculations for $\mathrm{NdFeO}_{3}$ yield similar magnetic moments of $4.5-4.7 \mu_{B}$ (depending on the $U$ value) that likewise exceed the experimental value of $4.18 \mu_{B}$ at $1.5 \mathrm{~K} .^{28}$

Magnetic couplings extracted from the DFT $+U$ band structure indicate a predominant interaction $J_{d}=191 \mathrm{~K}$ within the $\mathrm{Fe}_{2} \mathrm{O}_{7}$ dimer, in agreement with the $180^{\circ} \mathrm{Fe}-\mathrm{O}-\mathrm{Fe}$ exchange pathway, which is most favorable for the AFM coupling, according to the Goodenough-Kanamori-Anderson rules. Other nearest-neighbor couplings are also AFM, but much weaker than $J_{d}$ according to the lower $\mathrm{Fe}-\mathrm{O}-\mathrm{Fe}$ angles (see Table IV). In general, the couplings decrease as the $\mathrm{Fe}-\mathrm{O}-\mathrm{Fe}$ angle approaches $90^{\circ}$, although this trend is violated at the lowest angles $\left(J_{c 1}>J_{c 2}\right)$, where the direct $\mathrm{Fe}-\mathrm{Fe}$ exchange comes into play. The couplings beyond nearest neighbors are all below $2 \mathrm{~K}$ and can be safely neglected in a first approximation. The resulting spin lattice has pentagonal-like

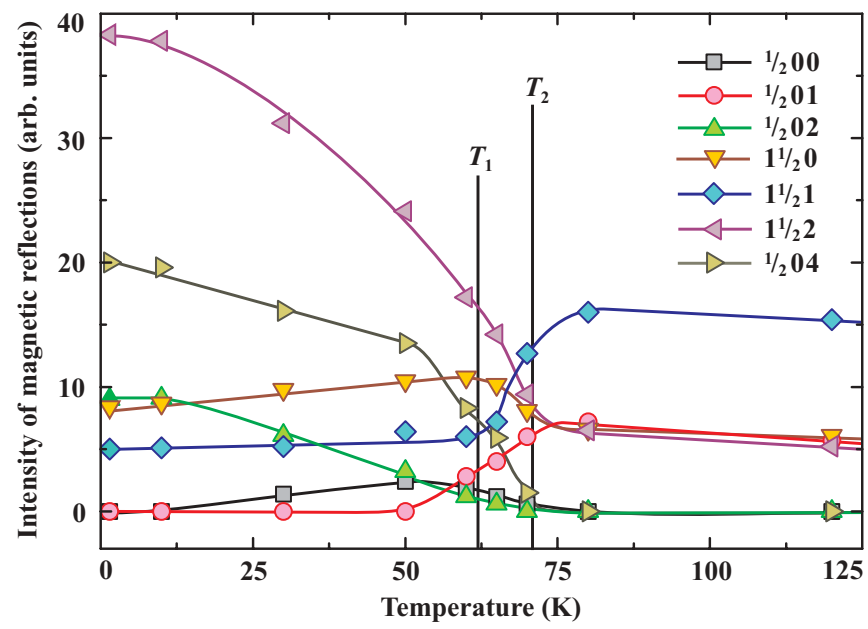

FIG. 6. (Color online) Temperature variation of intensity of magnetic reflections. 


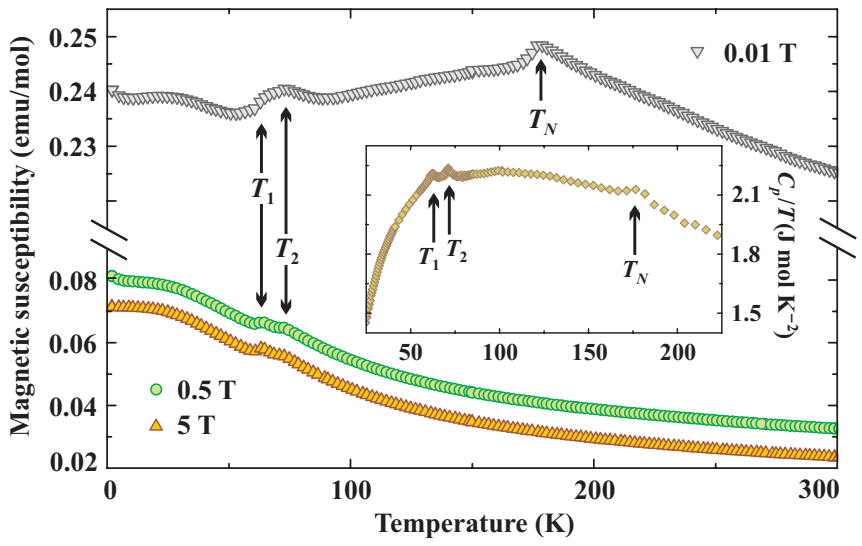

FIG. 7. (Color online) Magnetic susceptibility of $\mathrm{Bi}_{4} \mathrm{Fe}_{5} \mathrm{O}_{13} \mathrm{~F}$ measured in applied fields of $0.01,0.5$, and $5 \mathrm{~T}$. The sizable field dependence is due to a tiny amount of ferromagnetic impurity. The inset shows the heat capacity divided by temperature $\left(C_{p} / \mathrm{T}\right)$. Arrows denote the transition temperatures $T_{1}, T_{2}$, and $T_{N}$ (compare to Fig. 6).

units and three nonequivalent couplings in the $a b$ plane (Fig. 1, right). The interactions $J_{c 2}$ connect these planes along the $c$ direction via the $\mathrm{Fe} 3$ spins, which do not belong to the pentagonal units.

Monte Carlo simulations, performed for the spin lattice depicted in the right panel of Fig. 1 and the $J_{i}$ parameters from
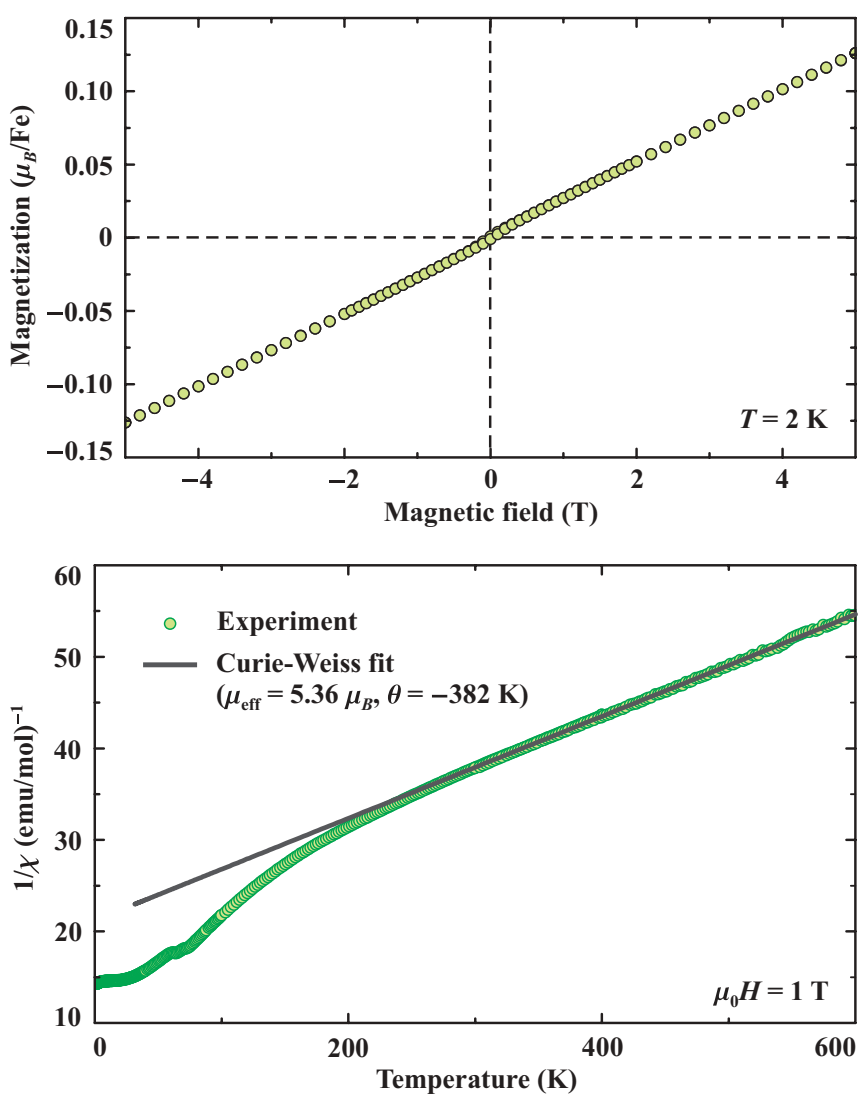

FIG. 8. (Color online) Magnetization curve of $\mathrm{Bi}_{4} \mathrm{Fe}_{5} \mathrm{O}_{13} \mathrm{~F}$ measured at $2 \mathrm{~K}$ (top) and inverse magnetic susceptibility (bottom) measured in the applied magnetic field of $1 \mathrm{~T}$ (circles) along with the Curie-Weiss fit with Eq. (2) (solid line).

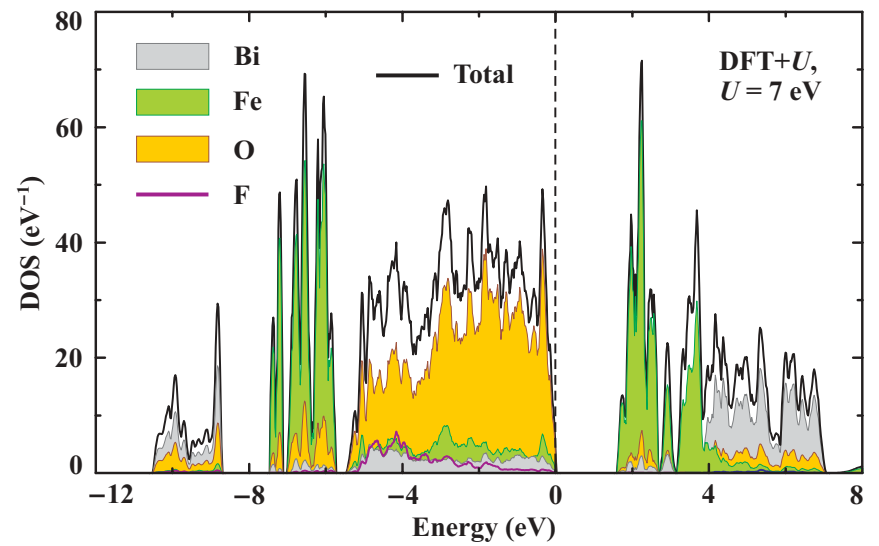

FIG. 9. (Color online) Electronic density of states calculated for the lowest-energy collinear AFM spin configuration of $\mathrm{Bi}_{4} \mathrm{Fe}_{5} \mathrm{O}_{13} \mathrm{~F}$ with DFT $+U(U=7 \mathrm{eV})$. The Fermi level is at zero energy. Only one spin direction is shown.

Table IV, suggest that at low temperatures $\mathrm{Bi}_{4} \mathrm{Fe}_{5} \mathrm{O}_{13} \mathrm{~F}$ should be magnetically ordered. The ordering pattern can be extracted from the spin-spin correlations (Table IV), as explained in Ref. 30. The normalized correlation $\left\langle S_{i} S_{j}\right\rangle / S^{2}$ is the measure of $\cos \varphi$, where $\varphi$ is the angle between the magnetic moments. Negative normalized correlations approaching -1 indicate antiparallel magnetic moments within the $\mathrm{Fe}_{2} \mathrm{O}_{7}$ dimers (the interaction $J_{d}$ ) as well as between the neighboring $\mathrm{Fe} 1$ and $\mathrm{Fe} 3$ along the $c$ direction (the interaction $J_{c 2}$ ). Despite the sizable AFM coupling $J_{c 1}$ between the adjacent Fe1 sites along $c$, the moments on these Fe1 sites are parallel, as shown by the positive spin-spin correlation of nearly +1 (Table IV). In this way, the simulations identify the ferrimagnetic order within the rutile-like chains, in agreement with the experiment.

The Monte Carlo simulations also confirm the non-collinear ordering pattern in the $a b$ plane. The spin-spin correlations of -0.81 and -0.84 for $J_{a b 1}$ and $J_{a b 2}$, respectively, imply that the magnetic moments on the adjacent Fe1 and Fe2 sites form an angle of about $145^{\circ}$, in agreement with the roughly $135^{\circ}$ arrangement observed experimentally. Altogether, our microscopic model of magnetic interactions in $\mathrm{Bi}_{4} \mathrm{Fe}_{5} \mathrm{O}_{13} \mathrm{~F}$ is in excellent agreement with the experimental ground-state magnetic configuration.

Following the expectations, the spin lattice of $\mathrm{Bi}_{4} \mathrm{Fe}_{5} \mathrm{O}_{13} \mathrm{~F}$ features pentagonal units with exclusively AFM interactions (Fig. 1, right) that lead to a strong magnetic frustration. Indeed, in the ground-state magnetic structure only the strongest

TABLE IV. Magnetic interactions in $\mathrm{Bi}_{4} \mathrm{Fe}_{5} \mathrm{O}_{13} \mathrm{~F}$. The table lists $\mathrm{Fe}-\mathrm{Fe}$ distances, $\mathrm{Fe}-\mathrm{O}-\mathrm{Fe}$ angles, exchange integrals $J_{i}$, as well as normalized spin-spin correlations $\left\langle S_{i} S_{j}\right\rangle / S^{2}$ in the ground-state magnetic configuration, as obtained from Monte Carlo simulations.

\begin{tabular}{lcccc}
\hline \hline Interaction & $d_{\mathrm{Fe}-\mathrm{Fe}}(\AA)$ & $\varphi_{\mathrm{Fe}-\mathrm{O}-\mathrm{Fe}}(\mathrm{deg})$ & $J_{i}(\mathrm{~K})$ & $\left\langle S_{i} S_{j}\right\rangle / S^{2}$ \\
\hline$J_{c 1}$ & 2.91 & 94.2 & 34 & +0.99 \\
$J_{c 2}$ & 3.06 & 97.4 & 10 & -0.94 \\
$J_{a b 1}$ & 3.39 & 119.2 & 45 & -0.84 \\
$J_{a b 2}$ & 3.53 & 130.9 & 74 & -0.80 \\
$J_{d}$ & 3.64 & 180 & 191 & -0.97 \\
\hline \hline
\end{tabular}


coupling $J_{d}$ is fully satisfied. The moments coupled by $J_{a b 1}$ and $J_{a b 2}$ develop the non-collinear configuration, whereas the AFM coupling $J_{c 1}$ is fully suppressed and gives way to the ferromagnetic (FM) order. The coupling $J_{c 2}$ is also fully satisfied, because it does not compete with any other interaction in this system.

The analysis of the Heisenberg Hamiltonian [Eq. (1)] determines the relative spin arrangement only. The arrangement of spins with respect to the crystallographic directions depends on magnetic anisotropy terms that should be addressed in future studies. While the planar spin arrangement is induced by the very nature of isotropic exchange couplings on the Cairo lattice, ${ }^{11}$ the location of spins in the $a b$ plane may be traced back to the tetragonal symmetry of the $\mathrm{Bi}_{4} \mathrm{Fe}_{5} \mathrm{O}_{13} \mathrm{~F}$ structure.

\section{DISCUSSION AND SUMMARY}

Our study puts forward $\mathrm{Bi}_{4} \mathrm{Fe}_{5} \mathrm{O}_{13} \mathrm{~F}$ as an interesting material prototype of the Cairo pentagonal spin lattice. A comprehensive structural characterization reveals the robust tetragonal symmetry and only minor structural changes between 1.5 and $700 \mathrm{~K}$. The rigid crystal structure ensures temperature-independent magnetic couplings and a reliable mapping of magnetic properties on the Cairo-lattice spin model.

Similar to $\mathrm{Bi}_{2} \mathrm{Fe}_{4} \mathrm{O}_{9}, \mathrm{Bi}_{4} \mathrm{Fe}_{5} \mathrm{O}_{13} \mathrm{~F}$ is not a perfect prototype of the Cairo spin lattice (Fig. 1). In $\mathrm{Bi}_{4} \mathrm{Fe}_{5} \mathrm{O}_{13} \mathrm{~F}$, the leading AFM coupling $J_{d}$ stands for the coupling $J_{1}$, whereas two nonequivalent couplings $J_{a b 1}$ and $J_{a b 2}$ stand for the single coupling $J_{2}$. Additionally, the lattice is decorated by Fe1 dumbbells that replace all four-vertex sites. However, this kind of decoration does not change any basic features of the magnetic system. The spin lattices of $\mathrm{Bi}_{2} \mathrm{Fe}_{4} \mathrm{O}_{9}$ and $\mathrm{Bi}_{4} \mathrm{Fe}_{5} \mathrm{O}_{13} \mathrm{~F}$ are topologically equivalent to the Cairo lattice. The non-collinear magnetic structures observed in these compounds follow theoretical predictions for the ideal Cairo lattice. ${ }^{15}$

Despite the similar magnetic ground state, $\mathrm{Bi}_{4} \mathrm{Fe}_{5} \mathrm{O}_{13} \mathrm{~F}$ is largely different from its $\mathrm{Bi}_{2} \mathrm{Fe}_{4} \mathrm{O}_{9}$ sibling. The latter compound undergoes a single magnetic transition, the AFM ordering at $T_{N}=238 \mathrm{~K}$. Below $T_{N}$, both magnetic susceptibility ${ }^{15}$ and magnetic neutron scattering ${ }^{15,31}$ show smooth temperature evolution, which is typical for a conventional antiferromagnet. In $\mathrm{Bi}_{4} \mathrm{Fe}_{5} \mathrm{O}_{13} \mathrm{~F}$, the magnetic ordering at $T_{N}=178 \mathrm{~K}$ is followed by two additional magnetic transitions that are accompanied by a sizable release of entropy (Sec. IIIC). Therefore, the AFM ordering that sets in below $T_{N}=178 \mathrm{~K}$ is likely incomplete and changes upon further magnetic transitions at $T_{1}$ and $T_{2}$. As none of these transitions is accompanied by any structural transformations detectable by NPD and SXPD (Sec. III A), the sizable release of entropy and the indications for a weak first-order contribution imply that above $T_{1}$ the magnetic order in $\mathrm{Bi}_{4} \mathrm{Fe}_{5} \mathrm{O}_{13} \mathrm{~F}$ is altered. One plausible explanation is the formation of partially disordered AFM states that have higher entropy than the fully ordered noncollinear AFM structure. The partially disordered states are found in magnetic models based on triangular spin lattices. ${ }^{32-34}$ They are indeed observed in $\mathrm{Co}^{2+}$ halides, such as $\mathrm{CsCoCl}_{3}{ }^{35}$ and $\mathrm{RbCoBr}_{3} .{ }^{36}$ Surprisingly, our experimental results may put forward similar physics on the pentagonal spin lattice.

Microscopically, the main difference between $\mathrm{Bi}_{2} \mathrm{Fe}_{4} \mathrm{O}_{9}$ and $\mathrm{Bi}_{4} \mathrm{Fe}_{5} \mathrm{O}_{13} \mathrm{~F}$ pertains to the stacking of the pentagonal layers. While in $\mathrm{Bi}_{2} \mathrm{Fe}_{4} \mathrm{O}_{9}$ these layers are directly stacked on top of each other, $\mathrm{Bi}_{4} \mathrm{Fe}_{5} \mathrm{O}_{13} \mathrm{~F}$ features additional $\mathrm{Fe} 3$ sites between the pentagonal layers. The voids formed between the $\mathrm{Fe}_{3} \mathrm{O}_{6}$ octahedra are filled with the $\mathrm{Bi}_{4} \mathrm{~F}$ tetrahedra that stabilize the modified crystal structure. One can also devise more complex stacking sequences and expand the family of materials with Cairo spin lattices. Although the change in the stacking sequence has no direct influence on the in-plane physics, the distance between the pentagonal layers affects the dimensionality of the spin system. Additionally, even weak structural changes have strong effect on the magnetism, as shown by the tangible difference in the magnetic behavior of $\mathrm{Bi}_{4} \mathrm{Fe}_{5} \mathrm{O}_{13} \mathrm{~F}$ and $\mathrm{Bi}_{2} \mathrm{Fe}_{4} \mathrm{O}_{9}$. We hope that our study will stimulate further theoretical work on both Cairo-lattice compounds available so far. A detailed experimental investigation of intricate magnetic transitions in $\mathrm{Bi}_{4} \mathrm{Fe}_{5} \mathrm{O}_{13} \mathrm{~F}$ will also be insightful.

In summary, $\mathrm{Bi}_{4} \mathrm{Fe}_{5} \mathrm{O}_{13} \mathrm{~F}$ is an interesting material prototype of the frustrated Cairo spin lattice. At low temperatures, $\mathrm{Bi}_{4} \mathrm{Fe}_{5} \mathrm{O}_{13} \mathrm{~F}$ develops non-collinear antiferromagnetic order, which is anticipated for this magnetic model. However, the magnetic ordering is strongly altered at $T_{1} \approx 62 \mathrm{~K}$ and $T_{2} \approx$ $71 \mathrm{~K}$, even though the crystal structure is virtually unchanged. The transitions at $T_{1}$ and $T_{2}$ also involve a sizable increase in the magnetic entropy that may be tentatively ascribed to the transformation toward partially disordered antiferromagnetic states.

\section{ACKNOWLEDGMENTS}

J.H. and D.B. acknowledge support from the Research Foundation-Flanders, through project G.0184.09N. G.V.T. acknowledges the financial support from ERC Grant No. 246791-COUNTATOMS. A.T. was funded by Alexander von Humboldt Foundation and the Mobilitas grant of the ESF. A.T. is also grateful to Ioannis Rousochatzakis for drawing his attention to the physics of the Cairo spin lattice. We are grateful to the ESRF and PSI for granting the beam time. Experimental support of Andy Fitch at the ID31 beamline of ESRF is kindly acknowledged.

\footnotetext{
"artem.abakumov@ua.ac.be

†altsirlin@gmail.com

${ }^{1}$ R. Moessner and A. P. Ramirez, Phys. Today 59, 24 (2006).

${ }^{2}$ L. Balents, Nature (London) 464, 199 (2010).

${ }^{3}$ A. P. Ramirez, Ann. Rev. Mater. Sci. 24, 453 (1994).

${ }^{4}$ J. E. Greedan, J. Mater. Chem. 11, 37 (2001).
}

\footnotetext{
${ }^{5}$ A. Harrison, J. Phys.: Condens. Matter 16, S553 (2004).

${ }^{6}$ J. S. Gardner, M. J. P. Gingras, and J. E. Greedan, Rev. Mod. Phys. 82, 53 (2010).

${ }^{7}$ H. D. Zhou, E. S. Choi, G. Li, L. Balicas, C. R. Wiebe, Y. Qiu, J. R. D. Copley, and J. S. Gardner, Phys. Rev. Lett. 106, 147204 (2011).
} 
${ }^{8}$ J. G. Cheng, G. Li, L. Balicas, J. S. Zhou, J. B. Goodenough, C. Xu, and H. D. Zhou, Phys. Rev. Lett. 107, 197204 (2011).

${ }^{9}$ C. Castelnovo, R. Moessner, and S. L. Sondhi, Nature (London) 451, 42 (2008).

${ }^{10}$ A. Ralko, Phys. Rev. B 84, 184434 (2011).

${ }^{11}$ I. Rousochatzakis, A. M. Lauchli, and R. Moessner, Phys. Rev. B 85, 104415 (2012).

${ }^{12}$ M. Rojas, O. Rojas, and S. M. de Souza, Phys. Rev. E 86, 051116 (2012)

${ }^{13}$ N. Nhzeki and M. Z. Wachi, Z. Kristallogr. Kristallgeom. Kristallphys. Kristallchem. 127, 173 (1968).

${ }^{14}$ A. G. Tutov and V. N. Markin, Izv. Akad. Nauk USSR, Neorg. Mater. 6, 2014 (1970).

${ }^{15}$ E. Ressouche, V. Simonet, B. Canals, M. Gospodinov, and V. Skumryev, Phys. Rev. Lett. 103, 267204 (2009).

${ }^{16}$ A. Altomare, M. C. Burla, M. Camalli, B. Carrozzini, G. L. Cascarano, C. Giacovazzo, A. Guagliardi, A. G. G. Moliterni, G. Polidori, and R. Rizzi, J. Appl. Crystallogr. 32, 339 (1999).

${ }^{17}$ V. Petřiček, M. Dušek, and L. Palatinus, JANA2006, the Crystallographic Computing System (Institute of Physics, Praha, 2006).

${ }^{18}$ C. Koch, Ph.D. thesis, Arizona State University, 2002.

${ }^{19}$ C. Prescher, C. McCammon, and L. Dubrovinsky, J. Appl. Crystallogr. 45, 329 (2012).

${ }^{20}$ K. Koepernik and H. Eschrig, Phys. Rev. B 59, 1743 (1999).

${ }^{21}$ J. P. Perdew and Y. Wang, Phys. Rev. B 45, 13244 (1992).

${ }^{22}$ C. Spiel, P. Blaha, and K. Schwarz, Phys. Rev. B 79, 115123 (2009).

${ }^{23}$ See Supplemental Material at http://link.aps.org/supplemental/ 10.1103/PhysRevB.87.024423 for details on the $\mathrm{Bi}_{4} \mathrm{Fe}_{5} \mathrm{O}_{13} \mathrm{~F}$ structure analysis, parameters of the Rietveld refinement using NPD data at $T=1.5$ and $700 \mathrm{~K}$, atomic coordinates, ADPs and interatomic distances refined from NPD data at $T=1.5$ and $700 \mathrm{~K}$, experimental, calculated and difference SXPD $(T=10$, 298 , and $673 \mathrm{~K})$ and $\mathrm{NPD}(T=1.5,300$ and $700 \mathrm{~K})$ profiles after Rietveld refinement, a room-temperature Mössbauer spectrum of $\mathrm{Bi}_{4} \mathrm{Fe}_{5} \mathrm{O}_{13} \mathrm{~F}$, and symmetry operators of the $P_{C} 4_{2} / n$ magnetic space group.

${ }^{24}$ D. Dickson and F. J. Berry, Mössbauer spectroscopy (Cambridge University Press, Cambridge, 2005).

${ }^{25}$ F. Menil, J. Phys. Chem. Solids 46, 763 (1985).

${ }^{26}$ B. J. Campbell, H. T. Stokes, D. E. Tanner, and D. M. Hatch, J. Appl. Crystallogr. 39, 607 (2006).

${ }^{27}$ I. V. Nikolaev, H. D’Hondt, A. M. Abakumov, J. Hadermann, A. M. Balagurov, I. A. Bobrikov, D. V. Sheptyakov, V. Y. Pomjakushin, K. V. Pokholok, D. S. Filimonov, G. Van Tendeloo, and E. V. Antipov, Phys. Rev. B 78, 024426 (2008).

${ }^{28}$ W. Sławiński, R. Przeniosło, I. Sosnowska, and E. Suard, J. Phys.: Condens. Matter 17, 4605 (2005).

${ }^{29}$ F. M. Mostafa and H. Ptasiewicz-Bak, J. Phys. Chem. Solids 47, 449 (1986).

${ }^{30}$ A. M. Abakumov, A. A. Tsirlin, J. M. Perez-Mato, V. Petřiček, H. Rosner, T. Yang, and M. Greenblatt, Phys. Rev. B 83, 214402 (2011).

${ }^{31}$ N. Shamir, E. Gurewitz, and H. Shaked, Acta Crystallogr., Sect. A 34, 662 (1978).

${ }^{32}$ M. Mekata, J. Phys. Soc. Jpn. 42, 76 (1977).

${ }^{33}$ M. Kaburagi, T. Tonegawa, and J. Kanamori, J. Phys. Soc. Jpn. 51, 3857 (1982).

${ }^{34}$ K. Wada and T. Ishikawa, J. Phys. Soc. Jpn. 52, 1774 (1983).

${ }^{35}$ M. Mekata and K. Adachi, J. Phys. Soc. Jpn. 44, 806 (1978).

${ }^{36}$ Y. Nishiwaki, T. Nakamura, A. Oosawa, K. Kakurai, N. Todoroki, N. Igawa, Y. Ishii, and T. Kato, J. Phys. Soc. Jpn. 77, 104703 (2008). 